\title{
The predictive prognostic values of serum interleukin-2, interleukin-6, interleukin-8, tumor necrosis factor- $\alpha$, and procalcitonin in surgical intensive care unit patients
}

\author{
Yamin Yan", Yan Hü, Xiaorong Wang, Zhenghong Yu, Yingjia Tang, Yuxia Zhang, Wenyan Pan \\ Nursing Department, Zhongshan Hospital, Fudan University, Shanghai, China \\ Contributions: (I) Conception and design: W Pan, Y Zhang; (II) Administrative support: W Pan, Y Zhang; (III) Provision of study materials or \\ patients: Y Yan, Y Hu; (IV) Collection and assembly of data: X Wang; (V) Data analysis and interpretation: Z Yu, Y Tang; (VI) Manuscript writing: \\ All authors; (VII) Final approval of manuscript: All authors. \\ \#These authors contributed equally to this work. \\ Correspondence to: Wenyan Pan, BN; Yuxia Zhang, PhD. Nursing Department, Zhongshan Hospital, Fudan University, NO.180, Fenglin Road, Xuhui \\ District, Shanghai 200032, China. Email: pan.wenyan@zs-hospital.sh.cn; zhang.yuxia@zs-hospital.sh.cn.
}

Background: The pathophysiological roles of serum cytokine levels in critically ill surgical patients has yet to be determined. This study aimed to determine the predictive prognostic values of serum interleukin IL-2, IL-6, IL-8, tumor necrosis factor- $\alpha(\mathrm{TNF}-\alpha)$, and procalcitonin (PCT) in surgical intensive care unit (ICU) patients.

Methods: Cytokine concentrations were measured with an IMMULITE 1000 Immunoassay System (Siemens Healthcare Diagnostics GmbH, Berlin, Germany). The study population was divided into quartiles according to the patients' cytokine levels: Q1, Q2, Q3, and Q4. The optimal cutoff values of IL-2, IL6 , IL-8, TNF- $\alpha$, and PCT level for predicting mortality were established by drawing receiver operating characteristic curves.

Results: The levels of IL-2 in Q3 [odds ratio $(\mathrm{OR})=4.434,95 \%$ confidence intervals (95\% CI): $1.527-$ 12.874] and $\mathrm{Q} 4$ (OR $=7.715$, 95\% CI: 2.744-21.693) were significantly higher than those in the Q1. The same results were noted in IL-6 and IL-8, and only Q4 (OR =2.383, 95\% CI: 1.419-4.001) showed significance in the level of TNF- $\alpha$. For IL-2, a cutoff value of $930.5 \mathrm{U} / \mathrm{mL}$ yielded a sensitivity of $69.39 \%$ and a specificity of $80.16 \%$ for the prediction of clinical outcome [area under the curve (AUC): $0.822 ; 95 \%$ CI: $0.789-0.855]$. For IL-6, a cutoff value of $50.95 \mathrm{pg} / \mathrm{mL}$ showed discrimination ability, yielding a sensitivity of $71.43 \%$ and a specificity of $61.75 \%$ for (AUC: 0.704 ; $95 \%$ CI: $0.660-0.748$ ). For IL-8, a cutoff value of $44.1 \mathrm{pg} / \mathrm{mL}$ yielded a sensitivity of $57.82 \%$ and a specificity of $79.58 \%$ for predicting clinical outcome (AUC: 0.753; 95\% CI: $0.713-0.793)$. For TNF- $\alpha$, a cutoff value of $11.95 \mathrm{pg} / \mathrm{mL}$ yielded a sensitivity and specificity of $68.66 \%$ and $72.90 \%$, respectively, in predicting clinical outcome (AUC: $0.758 ; 95 \%$ CI: 0.717-0.800). The positive likelihood ratios for IL-2, IL-6, IL-8, and TNF- $\alpha$ were 3.50, 1.87, 2.83 and 2.53, and the negative likelihood ratios were $0.38,0.46,0.53$, and 0.43 , respectively.

Conclusions: In critically ill patients, high levels of IL-2, IL-6, IL-8, and TNF- $\alpha$ in the first $24 \mathrm{~h}$ postoperatively were associated with clinical outcome. However, the effect of PCT level on prognosis still requires further study.

Keywords: Cytokine; predictive value; clinical outcome; critically ill patients

Submitted Aug 11, 2020. Accepted for publication Nov 09, 2020.

doi: 10.21037/atm-20-6608

View this article at: http://dx.doi.org/10.21037/atm-20-6608 


\section{Introduction}

Patients admitted to the intensive care unit (ICU) exhibit a high mortality rate regardless of the reason for admission. As well as providing useful information to improve therapeutic decision-making, the prediction of patient outcomes can help to guide the allocation of resources (1). However, the process of predicting morbidity and mortality in ICU patients following major surgery remains challenging. The extent to which levels of cytokines can inform the prediction of patient outcomes is still under debate.

Inflammatory response and immune system dysfunction have drawn an increasing amount of attention from researchers as important prognostic indicators $(2,3)$. In critically ill patients, dysfunction of the immune system is characterized by abnormal function and increased expression of pro-inflammatory cytokines, damageassociated molecular patterns (DAMPs) and acutephase proteins $(4,5)$. Habes et al. reported that a longer duration of extracorporeal circulation is associated with a more pronounced release of intestinal injury markers and the inflammatory cytokines interleukin (IL)- 6 and IL-8 (6). Cytokines can regulate host response, immune response, inflammation, and trauma. IL-6 is a dominant inflammatory cytokine in the postoperative period as well as an early predictor of sepsis. IL-6 can activate acute-phase response proteins, such as $\mathrm{C}$-reactive protein (CRP) and procalcitonin (PCT), along with negative feedback on IL-1 and tumor necrosis factor- $\alpha(\mathrm{TNF}-\alpha)$ production (7). IL-2 drives the growth, maturation, and differentiation of naive $\mathrm{T}$ cells into effector $\mathrm{T}$ cells (8), and directly affects immune function and local immune status (9).

Many studies have been conducted on inflammatory cytokines after surgery. Major surgery results in tissue injury, physical barriers, and potential exposure to environmental and commensal microbes, which can lead to local inflammation (7). Following surgery, excessive innate immune responses or the failure of adaptive immune responses induces the risk of significant morbidity and mortality from complications including inflammatory response syndrome (SIRS), infection, sepsis, and multiple organ dysfunction syndrome (MODS) (10,11).

Studies on cytokines related to major surgery have primarily examined fluctuations in the concentrations of cytokines. Dimopoulou et al. reported that the balance between TNF- $\alpha$ and IL-10 is associated with the occurrence of postoperative complications (12). Perioperative assessment of the levels of CRP, IL-6, IL-8, and IL-10 can help clinicians to predict and monitor postoperative atrial fibrillation (13). Various cytokines appear to be involved in the pathogenesis of systemic SIRS after major surgery (14); however, the pathophysiological process of their roles in the course of SIRS still needs to be explored.

In this study, we hypothesized that the serum levels of IL-2, IL-6, IL-8, TNF- $\alpha$, and PCT could serve as useful tools for predicting clinical outcomes in critically ill patients following major surgery. To test this hypothesis, we sought to detect the expression of IL-2, IL-6, IL-8, TNF- $\alpha$, and PCT, and to investigate the sensitivity and specificity of these cytokines for predicting mortality, in critically ill ICU patients after major surgery. Furthermore, we evaluated the prognostic significance of these cytokines to provide valuable information for the treatment of critically ill patients.

We present the following article in accordance with the STARD reporting checklist (available at http://dx.doi. org/10.21037/atm-20-6608).

\section{Methods}

\section{Setting and patients}

This retrospective study was conducted in a 50-bed surgery department and a 28-bed surgical ICU (SICU) in a teaching hospital (Clinical Trials Identifier: ChiCTR2000033894). The inclusion criteria were as follows: (I) consecutive adult patients; (II) patients undergoing elective or emergency major surgery; and (III) patients provided written informed consent. The exclusion criteria were: (I) a history of systemic inflammatory disease; (II) a history of glucocorticoid or cytokine/anticytokine therapy within the last year; or (III) baseline data or data on serum cytokine levels on SICU admission missing. Before the analysis, all patient data were anonymized and deidentified. All the patients received standard perioperative, anesthesia management and critical care.

This study was carried out retrospectively. All procedures performed in this study involving human participants were in accordance with the Declaration of Helsinki (as revised in 2013), and were in accordance with the standards of the Ethics Committee on Human Experimentation of Zhongshan Hospital, Fudan University (No. B2020-107R).

\section{Data collection}

Subjects were recruited and examined on the first day 
after admission to the SICU. The following data were collected: demographic information, including age and sex; admission diagnosis; surgical status and urgency (emergency or elective surgery); cytokine levels (IL-2, IL-6, IL-8, TNF- $\alpha$, and PCT), type of organ support [e.g., mechanical ventilation or continuous renal replacement therapy (CRRT)]. Between January 1, 2018, and June 30, 2019, data were collected daily by trained data collectors from the time of SICU admission to death or discharge from the SICU.

\section{Cytokine measurements}

Venous blood samples were obtained from the patients in the same time period (between 6:00 and 7:00 am) in the first $24 \mathrm{~h}$ postoperatively. Blood samples $(5 \mathrm{~mL})$ were collected into sterile tubes. After centrifugation, the serum was stored at $-80{ }^{\circ} \mathrm{C}$ until it was assayed. Serum concentrations of IL-2, IL-6, IL-8, TNF- $\alpha$, and PCT were measured with an IMMULITE 1000 Immunoassay System (Siemens Healthcare Diagnostics GmbH, Berlin, Germany), which uses chemiluminescent immunoassay technology. The normal ranges of detection were $223-710 \mathrm{U} / \mathrm{mL}$ for IL-2, $<3.4 \mathrm{pg} / \mathrm{mL}$ for IL-6, $<62 \mathrm{pg} / \mathrm{mL}$ for IL-8, $<8.1 \mathrm{pg} / \mathrm{mL}$ for TNF- $\alpha$, and $<0.5 \mathrm{ng} / \mathrm{mL}$ for PCT. The investigators who conducted these tests were blinded to all clinical data.

\section{Outcomes}

The primary outcome was hospital mortality. Secondary outcomes included: SICU readmission; length of SICU stay; tracheostomy; total days of organ support; receipt of mechanical ventilation at any time during SICU stay and the duration of mechanical ventilation; and receipt and duration of renal replacement therapy. Mechanical ventilation was defined as positive pressure ventilation via endotracheal tube or tracheostomy. Renal replacement therapy included all forms of continuous renal replacement therapy and intermittent hemodialysis.

\section{Statistical analysis}

Statistical analysis was performed using the SPSS 26.0 statistical package (SPSS Inc., Chicago, IL, USA). Categorical variables were presented as percentages, and continuous variables were presented as mean \pm standard deviation with normal distribution or median (interquartile range) with non-normal distribution. Average value was used to interpolate the missing data. The study population was divided into quartiles according to the patients' cytokine levels: Q1 (which also included untested cases), Q2, Q3, and Q4. Statistically significant differences between groups were assessed by chi-square for categorical variables, and by $t$-test or Mann-Whitney $\mathrm{U}$ test for continuous variables. The odds ratio (OR) and $95 \%$ confidence intervals $(95 \%$ CI) were calculated. Multivariate analyses were performed to determine independent risk factors for mortality, with adjustment for confounding variables according to the results of the univariate analysis. The optimal cutoff values of IL-2, IL-6, IL-8, TNF- $\alpha$, and PCT level for predicting mortality were established by drawing receiver operating characteristic (ROC) curves. A P value $<0.05$ indicated statistical significance.

\section{Results}

\section{Patient demographics and clinical characteristics}

This study included 5,120 patients, of whom 4,972 patients survived and 148 patients died, yielding a mortality rate of $2.9 \%$. The non-survivors were older than the survivors, with a mean age of $70.09 \pm 17.70$ years. The non-survivor group also had higher rates of SICU readmission, mechanical ventilation, CRRT, tracheotomy, and emergency surgery than the survivor group. The demographics and clinical characteristics of the surviving and non-surviving patients are shown in Table 1 .

\section{Cytokine levels in survivors and non-survivors}

Serum levels of IL-2, IL-6, IL-8, TNF- $\alpha$, and PCT in the non-survivors group were significantly higher than those in the survival group $(\mathrm{P}<0.001)$. The study population was divided into quartiles according to the patients' cytokine levels: Q1 (which also included untested cases), Q2, Q3, and Q4. Comparisons showed that the proportion of nonsurviving cases showed an increasing trend in each interval, while no significant trend was observed in the survival group (Table 2).

\section{Trend analysis of cytokine levels}

Serum levels of IL-2, IL-6, IL-8, TNF- $\alpha$, and PCT were fitted with logistic regression analysis in the form of grade variables and non-categorical variables, respectively. The results showed that the levels of IL-2, IL-6, IL-8, and $\mathrm{TNF}-\alpha$ had a significant linear correlation with patient 
Table 1 Baseline patient characteristics $(n=5,120)$

\begin{tabular}{|c|c|c|c|c|c|c|}
\hline Variables & Group & No. (\%) & \multicolumn{2}{|c|}{ Outcome } & $\chi^{2} / t / z$ & $P$ value \\
\hline \multirow[t]{2}{*}{ Gender } & Male & 3,065 (59.9) & $92(62.2)$ & 2,973 (59.8) & 0.335 & 0.563 \\
\hline & Female & $2,055(40.1)$ & $56(37.8)$ & $1,999(40.2)$ & & \\
\hline Age (years) & & & $70.09 \pm 17.70$ & $62.19 \pm 16.22$ & 5.839 & $<0.001$ \\
\hline \multirow[t]{3}{*}{ Emergency surgery } & Yes & $1,474(28.8)$ & $107(72.3)$ & $1,367(27.5)$ & 142.138 & $<0.001$ \\
\hline & No & 3,631 (70.9) & $40(27.0)$ & $3,591(72.2)$ & & \\
\hline & Unknown & $15(0.3)$ & $1(0.7)$ & $14(0.3)$ & & \\
\hline Mechanical ventilation & Yes & $1,182(23.1)$ & $132(89.2)$ & $1,050(21.1)$ & 375.053 & $<0.001$ \\
\hline CRRT & No & 4,859 (94.9) & $16(10.8)$ & 4,843 (97.4) & & \\
\hline \multirow[t]{2}{*}{ Tracheotomy } & Yes & $241(4.7)$ & $48(32.4)$ & $193(3.9)$ & 261.185 & $<0.001$ \\
\hline & No & $4,879(95.3)$ & $100(67.6)$ & $4,779(96.1)$ & & \\
\hline \multirow[t]{2}{*}{ SICU readmission } & Yes & $188(3.7)$ & $26(17.6)$ & $162(3.3)$ & 83.199 & $<0.001$ \\
\hline & No & 4,932 (96.3) & $122(82.4)$ & $4,810(96.7)$ & & \\
\hline
\end{tabular}

SICU, surgical intensive care unit; CRRT, continuous renal replacement therapy.

outcomes. The levels of IL-2 in Q3 (OR $=4.434,95 \% \mathrm{CI}$ : 1.527-12.874) and Q4 (OR =7.715, 95\% CI: 2.744-21.693) were significantly higher than those in Q1. The same results were observed for IL-6 and IL-8, and only group Q4 (OR $=2.383$, 95\% CI: $1.419-4.001)$ showed significant difference in the level of TNF- $\alpha$. No statistically significant difference was observed in PCT between the groups (Table 3).

\section{Predictive value of IL-2, IL-6, IL-8, and TNF- $\alpha$}

ROC curve analysis revealed that the area under the ROC curve of IL-2, IL-6, IL-8, and TNF- $\alpha$ was 0.822 , $0.704,0.753$, and 0.758 , respectively, indicating that these indicators carry diagnostic significance for patient outcomes. Take the maximum Youden index as the critical value, the optimal cutoff points for IL-2, IL-6, IL-8, and TNF- $\alpha$ were determined to be $930.5 \mathrm{U} / \mathrm{mL}, 50.95 \mathrm{pg} / \mathrm{mL}$, $44.1 \mathrm{pg} / \mathrm{mL}$, and $11.95 \mathrm{pg} / \mathrm{mL}$, respectively. Under these critical values, the sensitivity of IL-2, IL-6, IL- 8 and TNF- $\alpha$ was $69.39 \%, 71.43 \%, 57.82 \%$, and $68.66 \%$, respectively, and the specificity was $80.16 \%, 61.75 \%, 79.58 \%$ and $72.90 \%$, respectively. The positive likelihood ratios for IL-
2, IL-6, IL-8, and TNF- $\alpha$ were 3.50, 1.87, 2.83 and 2.53, respectively, and the corresponding negative likelihood ratios were $0.38,0.46,0.53$, and 0.43 , respectively (Table 4 and Figure 1).

\section{Discussion}

Surgical stress can influence cytokine levels, which reflected by the complexity of surgical procedure, the duration of the operation and the amount of blood transfused (12). Serum IL-2, IL-6, IL-8, TNF- $\alpha$, and PCT indirectly reflect the clinical severity status and the degree of organ system dysfunction. This study evaluated the levels of these cytokines in critically ill surgical patients. On the first postoperative day, IL-2, IL-6, IL-8, and TNF- $\alpha$ were observed to be significantly elevated in non-survivors, suggesting a potential correlation between high levels of these cytokines on the first postoperative day and an increased risk of mortality.

Accurate prognostic indicators of survival in ICU patients are important and helpful in guiding clinical decisionmaking (15). Currently, severity scores (such as Acute 
Table 2 Cytokine levels in survivors and non-survivors $(\mathrm{n}=5,120)$

\begin{tabular}{|c|c|c|c|c|c|}
\hline Cytokines group & No. (\%) & \multicolumn{2}{|c|}{ Outcome } & $\chi^{2} / t / z$ & $P$ value \\
\hline IL-2 & & $1,314.00(743.00,2,335.00)$ & $522.00(364.00,825.75)$ & -13.299 & $<0.001$ \\
\hline Q1 ( $\leq 367)$ & $1,292(25.2)$ & $4(2.7)$ & $1,288(25.9)$ & 188.681 & $<0.001$ \\
\hline Q2 (368-530) & $1,278(25.0)$ & $8(5.4)$ & $1,270(25.5)$ & & \\
\hline Q4 (>850) & $1,276(24.9)$ & $106(71.6)$ & $1,170(23.5)$ & & \\
\hline IL-6 & & $112.00(32.30,393.00)$ & $31.10(11.30,93.90)$ & -8.965 & $<0.001$ \\
\hline Q1 ( $\leq 11.50)$ & $1,295(25.3)$ & $9(6.1)$ & $1,286(25.9)$ & 69.717 & $<0.001$ \\
\hline Q2 (11.60-31.90) & $1,270(24.8)$ & $27(18.2)$ & $1,243(25.0)$ & & \\
\hline IL-8 & & $54.60(21.60,126.00)$ & $17.00(9.00,36.73)$ & -10.369 & $<0.001$ \\
\hline Q1 ( $\leq 9.00)$ & $1,390(27.1)$ & $8(5.4)$ & 1,382 (27.8) & 104.992 & $<0.001$ \\
\hline Q2 (9.10-17.00) & $1,194(23.3)$ & $18(12.2)$ & $1,176(23.7)$ & & \\
\hline Q3 (17.10-38.00) & $1,261(24.6)$ & $35(23.6)$ & $1,226(24.7)$ & & \\
\hline Q4 (>38.00) & $1,275(24.9)$ & $87(58.8)$ & $1,188(23.9)$ & & \\
\hline TNF- $\alpha$ & & $15.85(10.13,31.15)$ & $8.10(5.80,12.50)$ & -10.216 & $<0.001$ \\
\hline Q1 ( $\leq 5.90)$ & $1,633(31.9)$ & $23(1.4)$ & $1,610(32.4)$ & 105.881 & $<0.001$ \\
\hline Q2 (6.00-8.30) & 1,188 (23.2) & $10(6.8)$ & $1,178(23.7)$ & & \\
\hline Q3 (0.11-0.38) & $1,060(20.7)$ & 19 (12.8) & 1,041 (20.9) & & \\
\hline Q4 (>0.38) & $1,130(22.1)$ & $81(54.7)$ & $1,049(21.1)$ & & \\
\hline
\end{tabular}

IL, interleukin; TNF- $\alpha$, tumor necrosis factor- $\alpha$; PCT, procalcitonin.

Physiology and Chronic Health Evaluation (APACHE) and Sepsis-related Organ Failure Assessment(SOFA) scores), along with clinical and laboratory variables, are commonly used to track the function of multiple organ systems (16-19). Cytokines have been indicated to play important roles in host defense and the maintenance of tissue homeostasis; however, abnormal cytokine production can damage these functions, resulting in inflammation and tissue injury (20). Studies correlated the severity scores and inflammatory markers had aroused more interesting.
Presterl et al. (21) reported a correlation between the levels of CRP, IL-6, TNF, APACHE III, and mortality probability model II scores. Both scoring systems, as well as CRP, were significantly higher in non-survivors compared with survivors. Shimazui et al. (22) concluded that serum IL-6 was a more accurate predictor of the severity of organ dysfunction in critically ill patients than PCT or CRP, and rose more rapidly after the insult, reaching its peak earlier than SOFA score.

We found an increased rate of non-survivors 
Table 3 Logistic regression analysis of the relationships of IL-2, IL-6, IL-8, TNF- $\alpha$, and PCT with patient outcomes

\begin{tabular}{|c|c|c|c|c|c|}
\hline Variables & \multicolumn{4}{|c|}{ OR $(95 \% \mathrm{Cl})$} & $P$ value for trenc \\
\hline Model I: IL-2 & 1.000 & $1.264(0.365-4.384)$ & $4.434(1.527-12.874)$ & 7.715 (2.744-21.693) & $<0.001$ \\
\hline$P$ values & & 0.712 & 0.006 & $<0.001$ & \\
\hline Model II: IL-6 & 1.000 & $2.267(0.957-5.370)$ & 2.599 (1.126-5.999) & $3.820(1.709-8.538)$ & $<0.001$ \\
\hline Model III: IL-8 & 1.000 & $1.762(0.738-4.208)$ & 2.477 (1.118-5.488) & 4.241 (1.994-9.022) & $<0.001$ \\
\hline$P$ values & & 0.202 & 0.025 & $<0.001$ & \\
\hline Model IV: TNF- $\alpha$ & 1.000 & $0.683(0.320-1.456)$ & $1.446(0.800-2.611)$ & $2.383(1.419-4.001)$ & $<0.001$ \\
\hline$P$ values & & 0.324 & 0.222 & 0.001 & \\
\hline
\end{tabular}

Age, length of SICU stay, Mechanical ventilation, CRRT, Tracheotomy had been adjusted. IL, interleukin; TNF- $\alpha$, tumor necrosis factor- $\alpha$; $\mathrm{PCT}$, procalcitonin; SICU, surgical intensive care unit; CRRT, continuous renal replacement therapy.

Table 4 Predictive values of IL-2, IL-6, IL-8, and TNF- $\alpha$

\begin{tabular}{lcccc}
\hline Parameters & IL-2 & IL-6 & IL-8 & TNF- $\alpha$ \\
\hline AUC & $0.822(0.789-0.855)$ & $0.704(0.660-0.748)$ & $0.753(0.713-0.793)$ & $0.758(0.717-0.800)$ \\
Cutoff value & $930.5 \mathrm{U} / \mathrm{mL}$ & $50.95 \mathrm{pg} / \mathrm{mL}$ & $44.1 \mathrm{pg} / \mathrm{mL}$ & $11.95 \mathrm{pg} / \mathrm{mL}$ \\
Sensitivity & $69.39 \%$ & $71.43 \%$ & $57.82 \%$ & $68.66 \%$ \\
Specificity & $80.16 \%$ & $61.75 \%$ & $79.58 \%$ & $72.90 \%$ \\
+LR & 3.50 & 1.87 & 2.83 & 2.53 \\
-LR & 0.38 & 0.46 & 0.53 & 0.43 \\
\hline
\end{tabular}

+LR, positive likelihood ratio; -LR, negative likelihood ratio. IL, interleukin; TNF- $\alpha$, tumor necrosis factor- $\alpha$; PCT, procalcitonin; AUC, area under the curve.

in IL-2, IL-6, IL-8, and TNF- $\alpha$ from Q1 to Q4. IL-2 yielded an increased rate of non-survivors $(2.7 \%<5.4 \%<20.3 \%<71.6 \%)$; IL-6 yielded an increased rate of non-survivors $(6.1 \%<18.2 \%<23.6 \%<52.0 \%)$; IL- 8 yielded an increased rate of non-survivors $(5.4 \%<12.2 \%<23.6 \%<58.8 \%)$; TNF- $\alpha$ yielded an increased rate of non-survivors $(1.4 \%<6.8 \%<21.6 \%<56.1 \%)$; PCT yielded rate of $54.7 \%, 12.8 \%, 13.5 \%$, and $18.9 \%$ for Q4, Q3, Q2, and Q1, respectively. Our current data demonstrate a relationship between increased levels of IL-2, IL-6, IL-8, and TNF- $\alpha$ and mortality, which was consistent with previous studies $(12,23,24)$. Our findings show that IL2, IL-6, IL-8, TNF- $\alpha$, and to a lesser extent PCT, are of crucial importance in the outcomes of critically ill surgical patients.

From the trend analysis, significant differences were observed in IL-2, IL-6, IL-8, and TNF- $\alpha$ levels according to quartile [Q3 versus Q1 $(\mathrm{P}=0.006)$, and Q4 vs. Q1 $(\mathrm{P}<0001)$ in IL-2; Q3 vs. Q1 ( $\mathrm{P}=0.025)$, and $\mathrm{Q} 4$ vs. $\mathrm{Q} 1(\mathrm{P}=0001)$ in IL-6; Q3 vs. Q1 ( $\mathrm{P}=0.025)$, and $\mathrm{Q} 4$ vs. $\mathrm{Q} 1(\mathrm{P}<0001)$ in IL8; $\mathrm{Q} 4$ vs. Q1 ( $\mathrm{P}=0001)$ in TNF- $\alpha$ ]. No differences were found between the groups in relation to PCT, which has been widely used as a biomarker of bacterial infection (25). However, previous studies have reported that PCT is not an indicator of organ dysfunction (26), especially in severe patients, which could explain our result. Previous data on the cutoff values for these cytokines are inconsistent. Focusing on patients who underwent major abdominal 


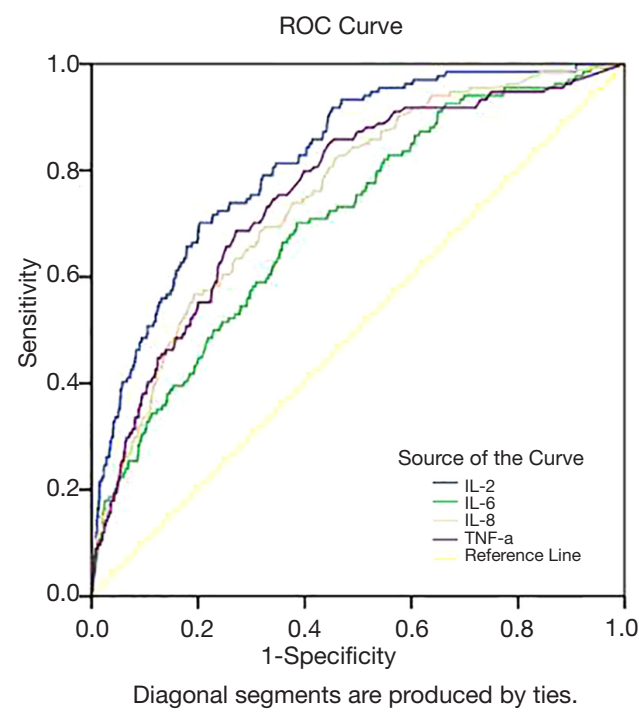

Figure 1 Receiver operating characteristic (ROC) curves showing the goodness of fit of the multivariable predictive model.

surgery, Rettig et al. (27) reported that IL-6 on the first day after surgery had the highest accuracy with a cutoff point of $432 \mathrm{pg} / \mathrm{mL}$; however, the optimal cutoff value in Mokart et al.'s study was $310 \mathrm{pg} / \mathrm{mL}$, as assessed using ROC curve analysis (28). Donati et al. (29) reported that the cutoff point for IL-8 was $60 \mathrm{pg} / \mathrm{dL}$. Meanwhile, for critically ill surgical patients, our results demonstrated that the optimal cutoff points for IL-2, IL-6, IL-8, and TNF- $\alpha$ were $930.5 \mathrm{U} / \mathrm{mL}$, $50.95 \mathrm{pg} / \mathrm{mL}, 44.1 \mathrm{pg} / \mathrm{mL}$, and $11.95 \mathrm{pg} / \mathrm{mL}$, respectively. It should note that the prognostic value of serum IL-2, IL6 , IL-8, TNF- $\alpha$, and PCT may be inconsistent in different diseases, the reason may related to the disease itself and the therapy, especially in cancer patients. The exact predictive value and cutoff points of these cytokines in different diseases still need further studies.

Our study has several limitations. Firstly, the cytokine levels were detected in the first $24 \mathrm{~h}$ after the patients were admitted to the SICU. As cytokine levels can be inhibited by immunosuppressive drugs such as steroids, this may have introduced bias to the results. Sequential and dynamic assessments may provide further evidence for the prediction of patients' clinical outcome. Secondly, this is a single-center retrospective study of critically ill surgical patients, and we did not analyze the associations between complications and cytokines. Multiple methods should be used to test the results in the future. Finally, CRRT may influence the level of cytokines.

\section{Conclusions}

Q4 in IL-2, IL-6, IL-8, TNF- $\alpha$, and PCT was associated with the highest mortality rate in the present study. Our results indicate that IL-2, IL-6, IL-8, and TNF- $\alpha$ have predictive value as biomarkers for mortality in critically ill surgical patients. However, the precise effect of PCT on clinical outcomes in SICU patients needs further investigation.

\section{Acknowledgments}

The authors sincerely thank Jian Gao for guiding the data collection and the statistical analysis for this study.

Funding: This work was supported by the Youth Program of Zhongshan Hospital, Fudan University (no. 2019ZSQN01), the Fuxing Nursing Program of Fudan University (no. FNF201945), and Program of Fudan University "Double First-Class Discipline Construction” (no. 2018-40-22).

\section{Footnote}

Reporting Checklist: The authors have completed the STARD reporting checklist.Available at http://dx.doi.org/10.21037/ atm-20-6608

Data Sharing Statement: Available at http://dx.doi. org/10.21037/atm-20-6608

Conflicts of Interest: All authors have completed the ICMJE uniform disclosure form (available at http://dx.doi. org/10.21037/atm-20-6608). The authors have no conflicts of interest to declare.

Ethical Statement: The authors are accountable for all aspects of the work in ensuring that questions related to the accuracy or integrity of any part of the work are appropriately investigated and resolved. This study was carried out retrospectively. All procedures performed in this study involving human participants were in accordance with the Declaration of Helsinki (as revised in 2013), and were in accordance with the standards of the Ethics Committee on Human Experimentation of Zhongshan Hospital, Fudan University (No. B2020-107R). Written informed consent was taken from patients.

Open Access Statement: This is an Open Access article distributed in accordance with the Creative Commons 
Attribution-NonCommercial-NoDerivs 4.0 International License (CC BY-NC-ND 4.0), which permits the noncommercial replication and distribution of the article with the strict proviso that no changes or edits are made and the original work is properly cited (including links to both the formal publication through the relevant DOI and the license). See: https://creativecommons.org/licenses/by-nc-nd/4.0/.

\section{References}

1. Ferreira FL, Bota DP, Bross A, et al. Serial evaluation of the SOFA score to predict outcome in critically ill patients. JAMA 2001;286:1754-8.

2. Hsing CH, Wang JJ. Clinical implication of perioperative inflammatory cytokine alteration. Acta Anaesthesiol Taiwan 2015;53:23-8.

3. Kaufmann KB, Heinrich S, Staehle HF, et al. Perioperative cytokine profile during lung surgery predicts patients at risk for postoperative complications-A prospective, clinical study. PLoS One 2018;13:e0199807.

4. Singer M, Deutschman CS, Seymour CW, et al. The Third International Consensus Definitions for Sepsis and Septic Shock (Sepsis-3). JAMA 2016;315:801-10.

5. Krychtiuk KA, Ruhittel S, Hohensinner PJ, et al. Mitochondrial DNA and Toll-Like Receptor-9 Are Associated With Mortality in Critically Ill Patients. Crit Care Med 2015;43:2633-41.

6. Habes QLM, Linssen V, Nooijen S, et al. Markers of Intestinal Damage and their Relation to Cytokine Levels in Cardiac Surgery Patients. Shock 2017;47:709-14.

7. Alazawi W, Pirmadjid N, Lahiri R, et al. Inflammatory and Immune Responses to Surgery and Their Clinical Impact. Ann Surg 2016;264:73-80.

8. Silk AW, Margolin K. Cytokine Therapy. Hematol Oncol Clin North Am 2019;33:261-74.

9. Huang C, Zhou L, Chang X, et al. B7-H3, B7-H4, Foxp3 and IL-2 expression in cervical cancer: Associations with patient outcome and clinical significance. Oncol Rep 2016;35:2183-90.

10. Ni Choileain N, Redmond HP. Cell response to surgery. Arch Surg 2006;141:1132-40.

11. Huang H, Lu X, Yang H, et al. Acute kidney injury after associating liver partition and portal vein ligation for staged hepatectomy for hepatocellular carcinoma: two case reports and a literature review. Ann Transl Med 2019;7:795.

12. Dimopoulou I, Armaganidis A, Douka E, et al. Tumour necrosis factor-alpha (TNFalpha) and interleukin-10 are crucial mediators in post-operative systemic inflammatory response and determine the occurrence of complications after major abdominal surgery. Cytokine 2007;37:55-61.

13. Weymann A, Popov AF, Sabashnikov A, et al. Baseline and postoperative levels of C-reactive protein and interleukins as inflammatory predictors of atrial fibrillation following cardiac surgery: a systematic review and meta-analysis. Kardiol Pol 2018;76:440-51.

14. Sarbinowski R, Arvidsson S, Tylman M, et al. Plasma concentration of procalcitonin and systemic inflammatory response syndrome after colorectal surgery. Acta Anaesthesiol Scand 2005;49:191-6.

15. Yousef AA, Suliman GA. The predictive prognostic values of serum TNF-alpha in comparison to SOFA score monitoring in critically ill patients. Biomed Res Int 2013;2013:258029.

16. Akavipat $\mathrm{P}$, Thinkhamrop J, Thinkhamrop B, et al. Acute Physiology and Chronic Health Evaluation (Apache) Ii Score - the Clinical Predictor in Neurosurgical Intensive Care Unit. Acta Clin Croat 2019;58:50-6.

17. Yalcin M, Godekmerdan E, Tayfur K, et al. The APACHE II Score as a Predictor of Mortality After Open Heart Surgery. Turk J Anaesthesiol Reanim 2019;47:41-7.

18. Basile-Filho A, Lago AF, Menegueti MG, et al. The use of APACHE II, SOFA, SAPS 3, C-reactive protein/albumin ratio, and lactate to predict mortality of surgical critically ill patients: A retrospective cohort study. Medicine (Baltimore) 2019;98:e16204.

19. Korkmaz Toker M, Gulleroglu A, Karabay AG, et al. SAPS III or APACHE IV: Which score to choose for acute trauma patients in intensive care unit? Ulus Travma Acil Cerrahi Derg 2019;25:247-52.

20. Jordan SC, Choi J, Kim I, et al. Interleukin-6, A Cytokine Critical to Mediation of Inflammation, Autoimmunity and Allograft Rejection: Therapeutic Implications of IL-6 Receptor Blockade. Transplantation 2017;101:32-44.

21. Presterl E, Staudinger T, Pettermann M, et al. Cytokine profile and correlation to the APACHE III and MPM II scores in patients with sepsis. Am J Respir Crit Care Med 1997;156:825-32.

22. Shimazui T, Matsumura Y, Nakada TA, et al. Serum levels of interleukin-6 may predict organ dysfunction earlier than SOFA score. Acute Med Surg 2017;4:255-61.

23. Wischmeyer PE, Mintz-Cole RA, Baird CH, et al. Role of heat shock protein and cytokine expression as markers of clinical outcomes with glutamine-supplemented parenteral nutrition in surgical ICU patients. Clin Nutr 2020;39:563-73. 
24. Iwase S, Nakada TA, Hattori N, et al. Interleukin-6 as a diagnostic marker for infection in critically ill patients: A systematic review and meta-analysis. Am J Emerg Med 2019;37:260-5.

25. Wacker C, Prkno A, Brunkhorst FM, et al. Procalcitonin as a diagnostic marker for sepsis: a systematic review and meta-analysis. Lancet Infect Dis 2013;13:426-35.

26. Karlsson S, Heikkinen M, Pettila V, et al. Predictive value of procalcitonin decrease in patients with severe sepsis: a prospective observational study. Crit Care 2010;14:R205.

27. Rettig TC, Verwijmeren L, Dijkstra IM, et al.

Cite this article as: Yan Y, Hu Y, Wang X, Yu Z, Tang Y, Zhang Y, Pan W. The predictive prognostic values of serum interleukin-2, interleukin-6, interleukin-8, tumor necrosis factor- $\alpha$, and procalcitonin in surgical intensive care unit patients. Ann Transl Med 2021;9(1):56. doi: 10.21037/atm-206608
Postoperative Interleukin-6 Level and Early Detection of Complications After Elective Major Abdominal Surgery. Ann Surg 2016;263:1207-12.

28. Mokart D, Merlin M, Sannini A, et al. Procalcitonin, interleukin 6 and systemic inflammatory response syndrome (SIRS): early markers of postoperative sepsis after major surgery. Br J Anaesth 2005;94:767-73.

29. Donati A, Battisti D, Recchioni A, et al. Predictive value of interleukin 6 (IL-6), interleukin 8 (IL-8) and gastric intramucosal $\mathrm{pH}(\mathrm{pH}-\mathrm{i})$ in major abdominal surgery. Intensive Care Med 1998;24:329-35. 